\title{
Epoxide Compound
}

National Cancer Institute

\section{Source}

National Cancer Institute. Epoxide Compound. NCI Thesaurus. Code C475.

A cyclic ether compound with a three-atom ring containing oxygen linked to two carbons.

These rings are highly stressed and more reactive than a typical acyclic ether. 